\title{
Erratum: Antagonism of a PCP Drug Discrimination by Hallucinogens and Related Drugs ${ }^{\text {th }}$
}

In the paper, West WB, et al., Antagonism of a PCP Drug Discrimination by Hallucinogens and Related Drugs. Neuropsychopharmacology 22:618-625, 2000, one of the authors names was misspelled. Alice Lou should be Alice Luo. 